\title{
Temperature monitoring during tissue freezing using ultrasound speed measurements
}

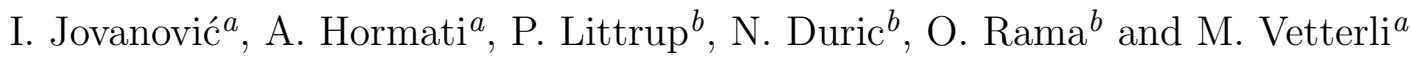 \\ ${ }^{a}$ Audiovisual Communications Laboratory, EPFL, CH-1015 Switzerland; \\ ${ }^{b}$ Karmanos Cancer Institute, 4100 John R. Street, WCCC, Detroit, MI
}

\begin{abstract}
A major limitation of thermal therapies is the lack of detailed thermal information needed to monitor the therapy. Temperatures are routinely measured invasively with thermocouples, but only sparse measurements can be made. Ultrasound tomography is an attractive modality for temperature monitoring because it is noninvasive, non-ionizing, convenient and inexpensive. It capitalizes on the fact that the changes in temperature cause the changes in sound speed. In this work we investigate the possibility of monitoring large temperature changes, in the interval from body temperature to $-40^{\circ} \mathrm{C}$. The ability to estimate temperature in this interval is of a great importance in cryosurgery, where freezing is used to destroy abnormal tissue. In our experiment, we freeze locally a tissue-mimicking phantom using a combination of one, two or three cryoprobes. The estimation of sound speed is a difficult task because, first, the sound is highly attenuated when traversing the frozen tissue; and second, the sound speed to be reconstructed has a high spatial bandwidth, due to the dramatic change in speed between the frozen and unfrozen tissue. We show that the first problem can be overcome using a beamforming technique. As the classical reconstruction algorithms inherently smooth the reconstruction, we propose to solve the second problem by applying reconstruction techniques based on sparsity.
\end{abstract}

Keywords: ultrasound tomography, temperature monitoring, cryosurgery, sparsity

\section{INTRODUCTION}

In this paper, we show how one can monitor temperatures during thermal therapies, more precisely during the cryosurgery, using ultrasound tomography. So far, the use of ultrasound for monitoring and guiding thermal therapies showed to be successful in more common reflection tomography method with B-mode scanners. ${ }^{1}$ Ultrasound transmission tomography, to the best of our knowledge, was not used for thermal therapy monitoring. We show that ultrasound transmission tomography offers a great potential for temperature monitoring and overcomes some of the problems related to the B-mode scanners.

Ultrasound tomography can provide a temperature monitoring modality that is non-invasive, non-interactive, non-ionizing, convenient and inexpensive. This modality is applicable if the temperature-dependent ultrasound parameters are: 1) identified, 2) measured, and 3) calibrated. It is shown that the sound speed, the attenuation and the backscattered energy are the most prominent temperature-dependent ultrasound parameters. In this work, we measure the changes in the sound speed induced by the temperature changes. Although we are not going to study the calibration, i.e., the sound speed versus temperature maps, we give an overview of the existing work, especially related to the breast tissues.

Further author information: (Send correspondence to I. Jovanović)

I. Jovanović: E-mail: ivana.jovanovic@epfl.ch, Telephone: +41 216931271

A. Hormati: E-mail: ali.hormati@epfl.ch, Telephone: +41 216937663

P. Littrup: E-mail: littrupp@karmanos.org, Telephone: +1 3135768757

N. Duric: E-mail: duric@karmanos.org, Telephone: +1 3135768706

O. Rama: E-mail: ramao@karmanos.org, Telephone: +1 3135768253

M. Vetterli: E-mail: martin.vetterli@epfl.ch, Telephone: +41 216935698

Medical Imaging 2009: Ultrasonic Imaging and Signal Processing, edited by Stephen A. McAleavey, Jan D'hooge Proc. of SPIE Vol. 7265, 72650Q - ( 2009 SPIE - CCC code: 1605-7422/09/\$18 - doi: 10.1117/12.813682 


\subsection{Sound speed changes with temperature changes in tissues}

In general, speed of sound has the following relationship to the coefficient of stiffness $C$, and the material density $\rho$ :

$$
c=\sqrt{\frac{C}{\rho}} .
$$

Both $C$ and $\rho$ depend on temperature in a non-linear fashion. Moreover, tissue represents a mixture of different materials and therefore the speed vs. temperature relationship needs to be examined individually for every tissue of interest.

In the past, many researchers experimentally measured the speed vs. temperature relationship for different tissue types. One of the first motivations was to provide thermal dosimetry for the treatment of cancer by hyperthermia, or by high temperature ablation, modalities which are now widely accepted by the medical community. It was found that, in water and in most tissue media, sound speed increases with increasing temperature, ${ }^{2}$ for temperatures above $0^{\circ} \mathrm{C}$. However, in fatty tissues, sound speed decreases with increasing temperature. ${ }^{2}$ For the case of breast tissue, it is shown in Ref. 3 that in non-fatty breast tissue the sound speed increases approximatively linearly from $1540 \mathrm{~m} / \mathrm{s}$ to $1580 \mathrm{~m} / \mathrm{s}$ in the temperature interval from $23^{\circ} \mathrm{C}$ to $43^{\circ} \mathrm{C}$. However, the slope for breast fat and fatty breast parenchyma becomes negative between about $32^{\circ} \mathrm{C}$ and $35^{\circ} \mathrm{C}$, and then it is positive for temperature values above $40^{\circ} \mathrm{C}$. It is hypothesized that the decrease in speed of sound of fatty breast tissue between $32^{\circ} \mathrm{C}$ and $35^{\circ} \mathrm{C}$ is due to the phase changes associated with melting of some components of breast fat.

Currently, there is a growing interest for monitoring the temperatures below $0{ }^{\circ} \mathrm{C}$, since many promising minimally invasive thermal therapies are based on freezing the cancerous cells. So far, little research is done to construct the speed vs. temperature maps for subzero temperatures. The expectation is that tissues behave similarly to water, in which case the sound speed increases abruptly below $0^{\circ} \mathrm{C}$, and continues to increase as the temperature decreases. This is confirmed in case of the skin tissue. It is found in Ref. 4 that the mean sound speed of the frozen pig skin is $2865 \pm 170 \mathrm{~m} / \mathrm{s}$.

\subsection{Cryosurgery}

Cryosurgery, sometimes referred to as cryotherapy or cryoablation, is a surgical technique that uses cytotoxic cold temperatures to destroy cancerous cells by direct cell injury from ice crystal formation. ${ }^{5}$ A coolant or cryogen is circulated through a cryoprobe (see Figure 1(a)) that is placed in contact with the tissue to be destroyed. The extraction of heat from the tissue at the cryoprobe tip produces a frozen region that penetrates into the tissue, forming a hemispheric freezing interface commonly called the iceball (see Figure 1(b)).

Developed first in the middle of the nineteenth century it has recently experienced a revival thanks to the development of imaging techniques used to monitor the cryosurgery. The authors in Ref. 1 worked on monitoring cryosurgery with conventional ultrasound. They showed that the discontinuity of the acoustic impedance from unfrozen to frozen tissue can be successfully imaged with B-mode scans. The accuracy of this image is limited by the assumption that the sound speed in the tissue is uniform and known. Because the ice reflects most of the energy, the area behind the freezing interface is in shade, and cannot be imaged. However, the temperature distribution inside the frozen region is also of importance since the cytotoxic tissue temperatures (i.e. $<-20^{\circ} \mathrm{C}$ ) appear only at some distance behind the leading edge of the visualized frozen region. So far, the temperatures inside the frozen region are interpolated based on experience on the temperature gradient observed in the previous experiments. ${ }^{6}$ If we imagine more complicated ice forms (not only the iceball), then it becomes difficult to predict the temperature distribution inside the frozen region. These drawbacks motivate the development of other techniques as MRI, optical imaging, electrical impedance tomography, for cryosurgery monitoring. MRI overcomes the acoustic shadowing but it is much more expensive and it requires special surgical tools and a special environment. Acoustic tomography offers a promising alternative for monitoring cryosurgery, since the region of interest is insonofied from many directions. Therefore, the shadowing effect almost disappear as it will be shown in the following. The technique is also inexpensive and easy to use. 


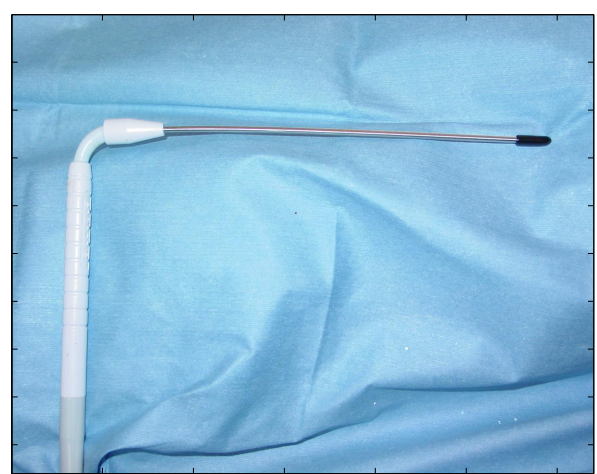

(a)

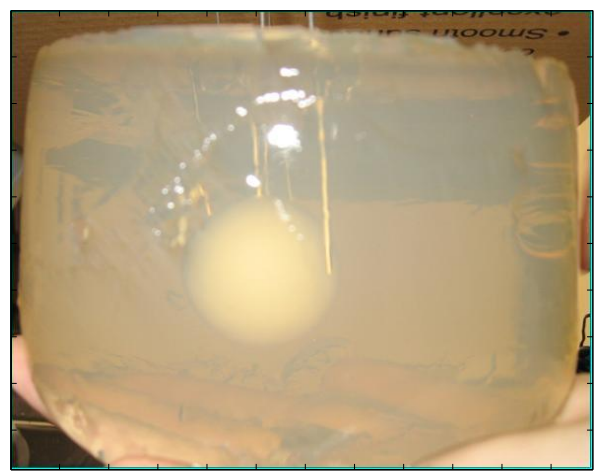

(b)

Figure 1. Cryosurgery is a minimally invasive thermal therapy. (a) An example of a cryoprobe. They exist in different sizes, needle diameters, and power. (b) The iceball formed inside the gelatine phantom with the diameter of approximatively $3 \mathrm{~cm}$.

\section{MATERIALS AND METHODS}

\subsection{Experimental setup}

In our experiment, the gelatine phantom is locally frozen using focal cooling induced by a freeze cycle around an available cryoprobe. We use 3 cryoprobes positioned in the form of a triangle with each cryoprobe being $30 \mathrm{~mm}$ away from the other two. The motivation for using the 3 cryoprobes was to show that acoustic tomography has the ability to image complex shapes of frozen regions. After the cryoprobes are powered on, the iceballs around the cryoprobes start to grow, and after $5-10$ minutes reach the size of approximately $2.0-3.0 \mathrm{~cm}$ in diameter. During this period we estimate the sound speed inside the phantom. The sound speed is monitored using the CURE device, that is a clinical prototype tomographic ultrasound scanner, designed at the Karmanos Cancer Institute (KCI), Detroit. A detailed description of the scanner is given in Ref. 7.

The CURE scanner consists of the 20-cm-diameter ring, equipped with 256 equally spaced transducers and immersed in a water tank. The ultrasound signal is sequentially transmitted by each element and subsequently received with all 256 elements. This sequence is then repeated for each transmitting element, taking approximately 0.1 seconds to acquire a full slice. The operating frequency is $1.5 \mathrm{MHz}$. The CURE scanner is primarily used for breast screening. The patient is positioned prone with the breast situated through a hole in the canvas bedding. The breast is suspended in the water tank, and encircled by the ring. The ring can translate in the vertical direction to image the entire breast volume.

In this experiment, we monitor the sound speed every 15 seconds. The ring setup with the gelatin phantom inside is shown in Figure 2.

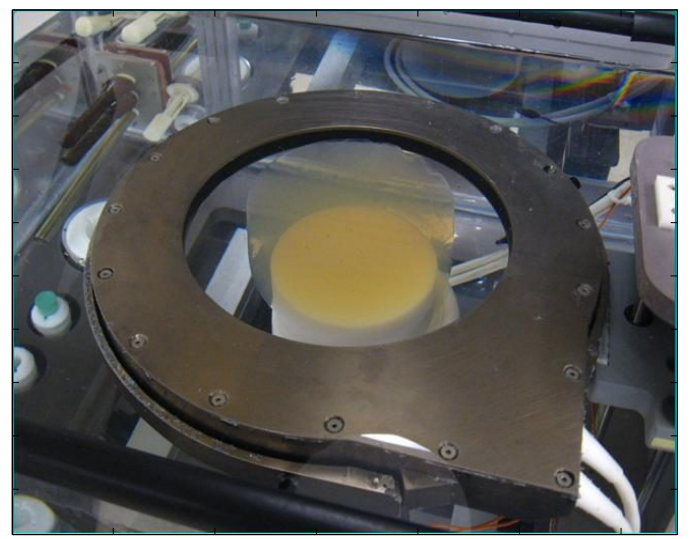

Figure 2. CURE temperature monitoring setup. A issue-mimicking phantom is immersed in water. 
The sound speed estimation inside frozen tissue showed to be a difficult task. It consists of two phases. In the first, i.e., the acquisition phase, we estimate the time-of-flight between every transmitter-receiver pair. In the second, i.e., the reconstruction phase, we use these time-of-flights and the known geometry of the setup to compute the sound speed inside the ring. The sound propagation is modeled using the bent ray theory, and an iterative algorithm is used to resolve the sound speeds. We identified two major problems, one related to the acquisition phase and the other related to the reconstruction phase.

\subsection{Time-of-flight estimation}

Time-of-flight estimation, which serves as the first stage that feeds into subsequent precessing blocks of a system has the most significant importance for the reconstruction. In the time-of-flight estimation problem, we can either estimate the absolute time-of-flight or the difference with respect to the arrival of any reference signal. The reference signal can be a signal sent through a known or controlled medium, for example water with a uniform known temperature. The correct time-of-flight will be then equal to the sum of the difference and the reference time-of-flight. Intuitively, estimating the difference of the time-of-flight is an easier problem because the systematic error that can appear when estimating the absolute time can be avoided. For example, the delays of the transducers, all electronic delays, and the transducers positions, although carefully taken into account, are never perfectly estimated and they are potential sources of the systematic error. However, the same systematic error appears in the time-of-flight of the reference signal, and it is going to be canceled when the difference between these two time-of-flights is computed. Whenever the specific problem nature offers the possibility of having the reference signal, its use can only improve the time-of-flight estimation.

In this application we use the reference signal through the water and we estimate the time-of-flight difference between that signal and the signal that goes through the ice-balls. The difference is computed by comparing the position of the "marker" point in the two signals. For the marker point we choose the first peak in the arriving signal. More on this method can be found in Ref. 8.

The major problem that we have in the time-of-flight estimation is due to the strong signal attenuation. Namely, upon formation of ice, the sound attenuation in the ice is dramatically increased allowing only a small percentage of the transmitted energy to go through the ice. Without help of signal processing tools needed to detect the signal that goes through the frozen part, we only receive the signal that travels on the surface of the iceballs. This signal is not attenuated, and as it travels on the surface of the iceball, where the sound speed is much lower than in the ice, it actually arrives later than the correct signal. As a result, the reconstructions with this signal showed that the sound speed in the frozen tissue decreases linearly (as the iceballs grow) with temperature. This was an obvious indicator that we did not estimate the correct time-of-flights. To increase the power of the received signal we use the beamforming technique.

Beamforming is a signal processing technique used with arrays of transmitters or receivers that control the directionality of a radiation pattern. When receiving a signal, the so called receive beamforming can increase the receiver sensitivity in the direction of wanted signals and decrease the sensitivity in the direction of interference and noise. When transmitting a signal, the so called transmit beamforming can increase the power along the chosen direction. Therefore, using beamforming we can enhance the signal in a particular direction and suppress noise and reflections coming from other directions. To this end, we use a simple delay and sum beamformer. This beamformer is quite true to its name as it merely takes the set of signals, delays and maybe weights them by varying amounts, and then adds them all together. The delays are determined by the direction (for farfield) or point (for nearfield) at which the array or transmitters or receivers is aimed. Despite its simplicity, delay and sum beamformer manages to achieve optimal noise suppression for the case of a point source in a background of white noise. The details on how to compute the correct delays of the beamformer are presented in our patent. ${ }^{9}$

\subsection{Sound speed reconstruction}

In the reconstruction phase, we have tried 3 different algorithms to invert the measurements back to the unknown sound speed. Our first choice is the solution that will merely minimize the $\ell_{2}$ norm of the residual. This will allow us to reconstruct the unknown sound speed which best matches the measurements. Since we deal with very high dimensional data, we decided to use the conjugate gradient algorithm to iteratively approach the solution. Note that this approach does not employ any prior knowledge about the unknown field. 
In the second and third algorithms, our goal is to include some prior knowledge in the reconstruction phase. Our emphasis is on the use of the methods based on sparsity. In this approach, the assumption is that the unknown object not only should satisfy the measurements, but also it should have sparse coefficients in some appropriate domain.

In our second algorithm, we assumed that the unknown field is sparse in the signal domain, i.e., there are a few dominant active cells while the rest contain no useful information. These active cells correspond to the position of the ice balls which have much higher sound speed with respect to the surrounding area. We implemented the matching pursuit (MP) ${ }^{10}$ sparse reconstruction algorithm. MP is an iterative, non-linear procedure which is used to decompose a signal into a linear expansion of waveforms chosen from a redundant dictionary. It starts by iteratively selecting the most active cell and subtracting its effect from the measurements. Then, it continues this procedure until either sufficient number of cells is selected or the residual drops below some specific threshold.

The third algorithm we employed is based on the sparsity assumption in the wavelet domain. It is well-known that wavelet transform is capable of representing well the piecewise smooth objects and therefore it should be able to represent well the ice-zone. We used the two dimensional daubechies-5 wavelet family in our algorithm. Because of the large size of the matrices, we had to choose a computationally tractable method. In this context, we minimized the $\ell_{1}$-penalized least square problem, using non-linear conjugate gradient descent algorithm with back-tracking line search ${ }^{11}$

$$
\underset{x}{\operatorname{argmin}}\|y-\Phi x\|_{2}+\lambda\|\Psi x\|_{1},
$$

where $\Phi$ is the measurement matrix, $\Psi$ is the sparsity basis and $\lambda$ determines the trade-off between data consistency and sparsity.

Upon formation of ice, the sound speed markedly increases $(\sim 2500 \mathrm{~m} / \mathrm{s})$ with respect to non-frozen tissue $(\sim 1500 \mathrm{~m} / \mathrm{s})$. The computed travel times confirm this fact. However, it is difficult to reliably reproduce the strong sound speed gradient that appears around the edge of the ice-zone using the classical tomography reconstruction methods. Therefore, although the travel time indicates a sudden and dramatic change of the sound speed the classical reconstruction is inherently smoothed. This problem is partially overcome with the use of the reconstruction algorithms based on sparsity.

\section{RESULTS}

The results show that it is possible to estimate a large range of sound speed variations within the tissue. Figure 3 represents the sound speed distribution 15 seconds after the three cryoprobes were switched on. The speed is estimated using the two inversion algorithms: the conjugate-gradient (CG) shown in Figure 5(a), and the matching pursuit (MP) shown in Figure 3(b). No sparsifying transform is applied in the second algorithm, and only the sparsity in the signal domain is assumed. The ice is formed and the sound speed increases abruptly. The regions of the high sound speed, that is in this case around $1800 \mathrm{~m} / \mathrm{s}$, correspond to the ice zones. Still, the speed is not as high as expected due to the inherent smoothing of the tomography methods. Higher reconstruction error concentrated at the edge of the region of interest, i.e. the ring, can be noticed in the CG method. Figure 4 shows the details of Figure 3 around the three cryoprobes. We can see that the MP method allows to increase the resolution of the image. The pixel size is $1.15 \times 1.15 \mathrm{~mm}^{2}$ for the MP method, and $2 \times 2 \mathrm{~mm}^{2}$ for the CG method. The reconstruction in Figure 4(b) is more localized, while in Figure 4(a) we have a significant blurring effect. Figure 5 shows the evolution of the iceballs over time using the CG method, the MP method, and also the method based on sparsity but in the wavelet domain. Specifically, for this example, we use the Daubechies wavelets family of the 7 th order.

We can follow the growth of the iceballs indicated by the abrupt increase of the sound speed in that region. For the comparison and the visualization purpose, the color scales are the same for all three methods at each time instant. We can notice that the best reconstruction is obtained by imposing sparsity in the wavelet domain. The CG and the MP methods obtain similar results, but looking at the details (see Figure 4) the MP algorithm still performs better. When the iceballs are big, the received signal is very attenuated and the iceballs are losing the exact shape in the CG and MP method (see Figure 5(j) and figure $5(\mathrm{k})$ ), but the wavelet based sparsity method still gives a good result (see Figure 5(l)). 


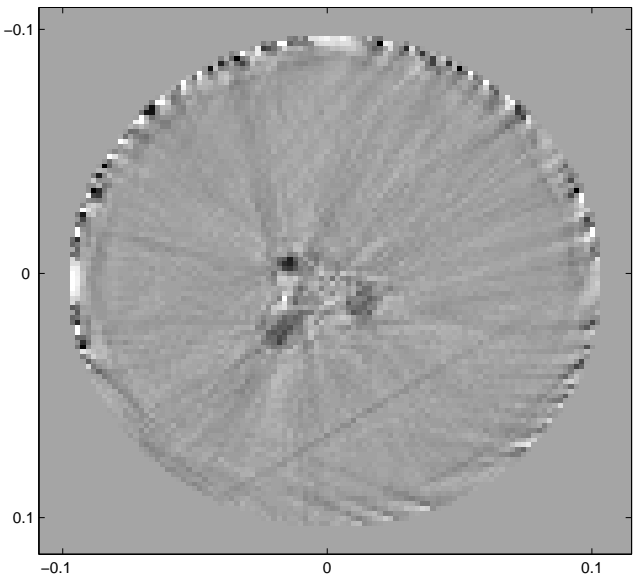

(a)

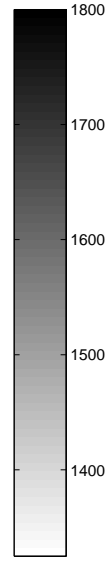

1500

1400

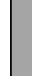

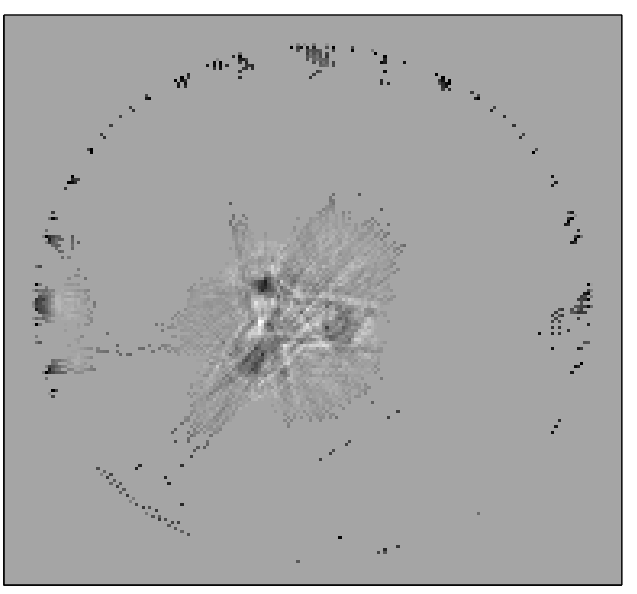

(b)

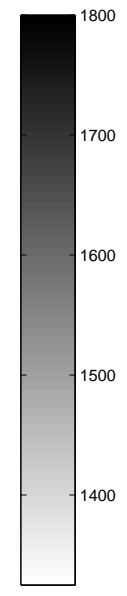
Figure 3. Sound speed estimation 15 seconds after the three cryoprobes are switched on. (a) The reconstruction using
the conjugate-gradient method. (b) The reconstruction obtained using the inversion based on sparsity, with the matching pursuit algorithm. This method allows to increase the resolution of the image.

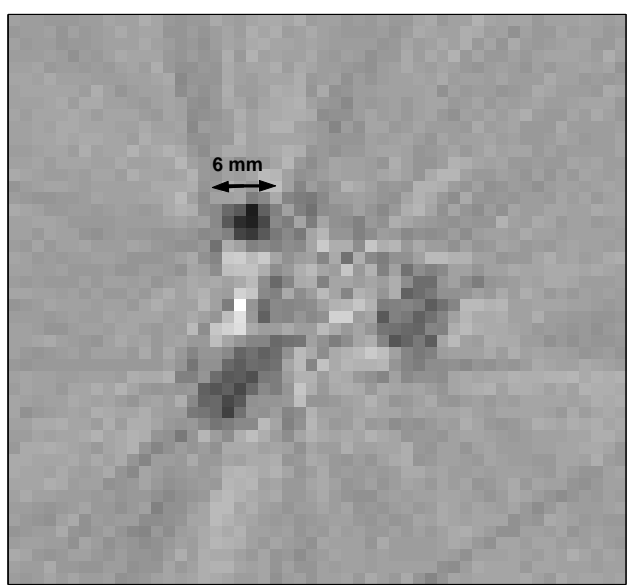

(a)
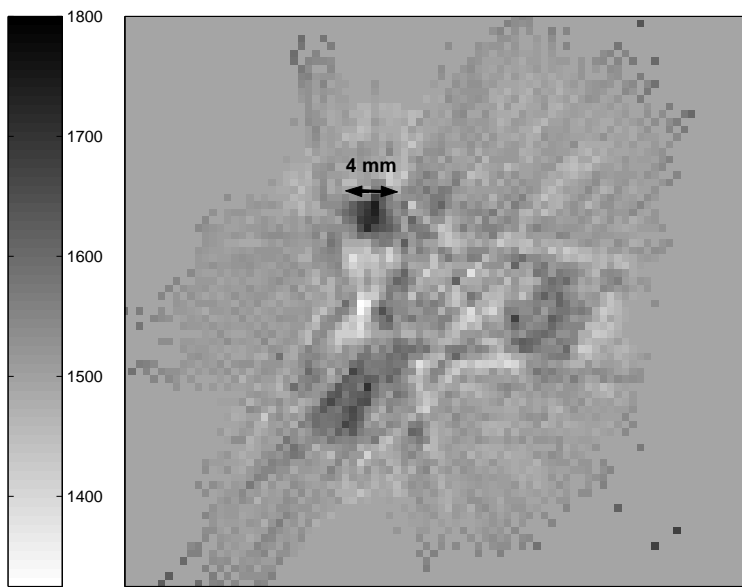

(b)

Figure 4. Detail of Figure 3. (a) The reconstruction using the conjugate-gradient algorithm. Detail of Figure 3(a). (b) The reconstruction using the algorithm based on sparsity. Detail of Figure 3(b). 


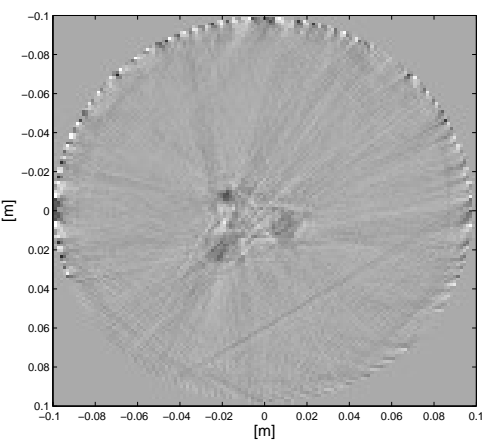

(a)

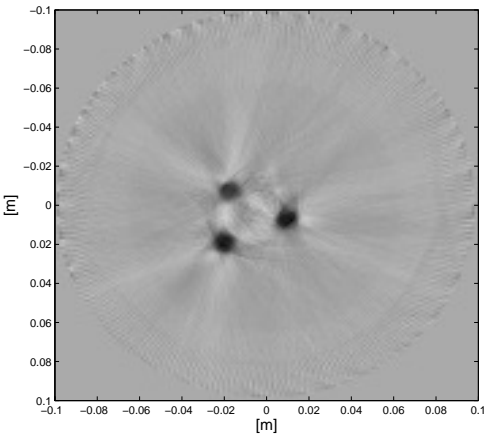

(d)

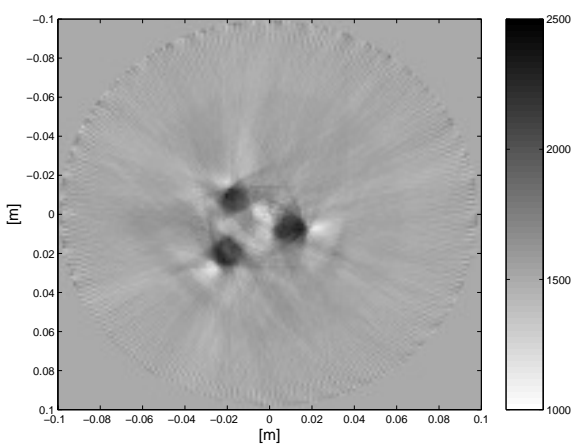

(g)

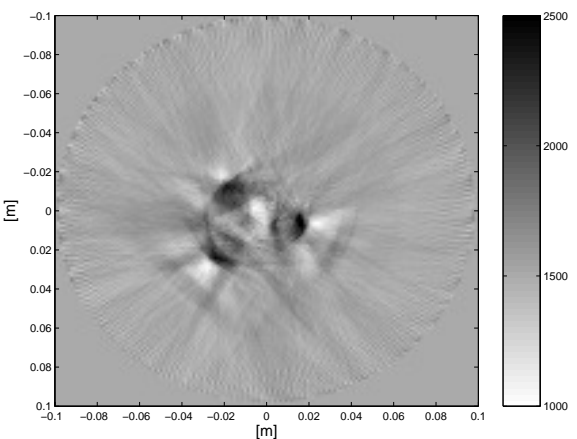

(j)

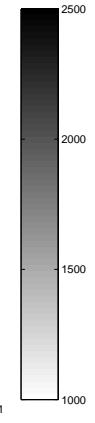

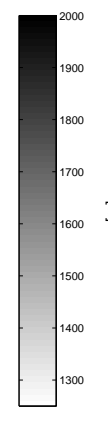

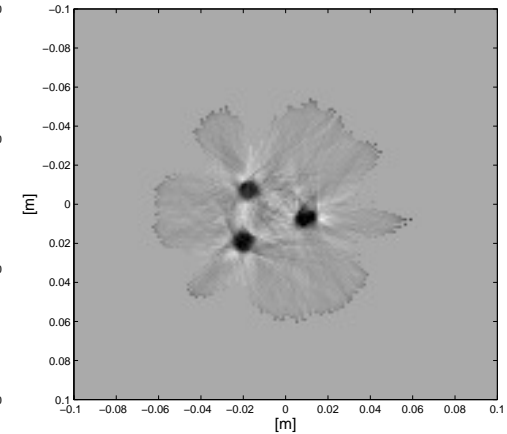

(e)

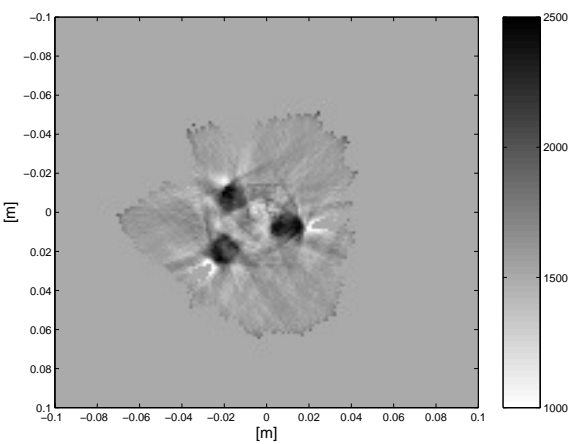

(h)

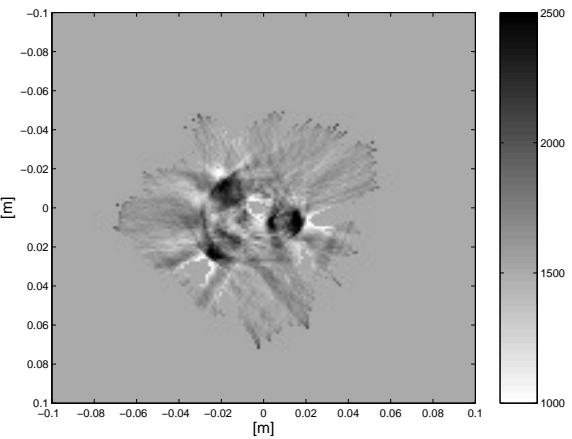

(k)

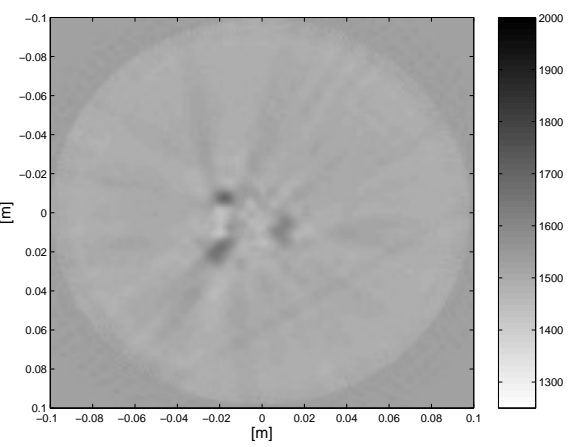

(c)

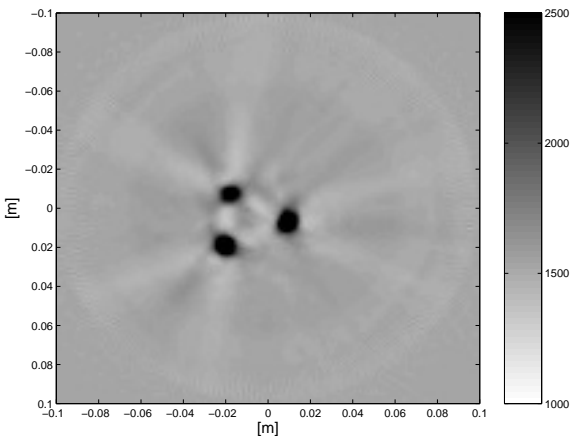

(f)

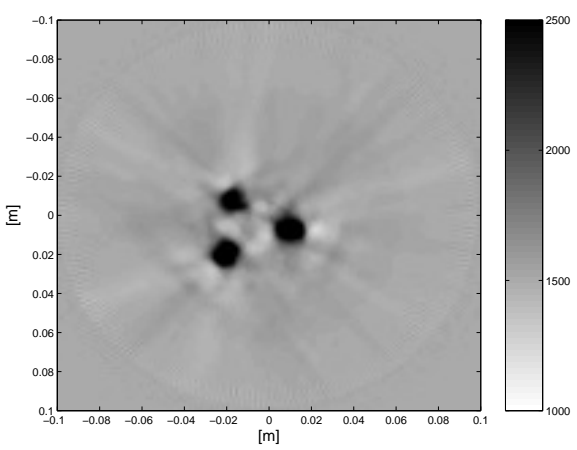

(i)

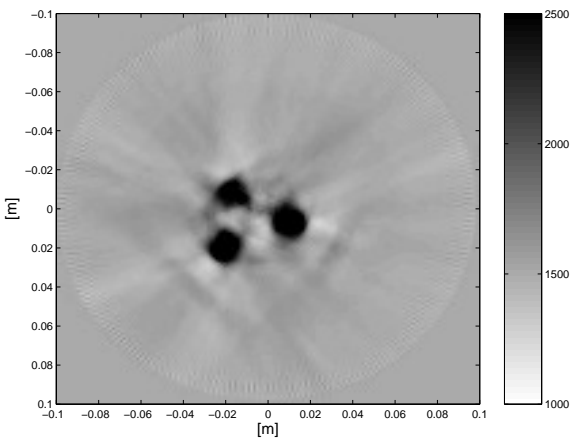

(I)

Figure 5. Sound speed estimation over time. The iceballs are growing over time, and the sound speed increases as the temperature decreases. The three reconstruction algorithms are compared at each time instant. 15 s: (a) the CG method, (b) the MP method, (c) the wavelet based sparsity method; 1 min: (d) the CG method, (e) the MP method, (f) the wavelet based sparsity method; 1 min $45 \mathrm{~s}$ : (g) the CG method, (h) the MP method, (i) the wavelet based sparsity method; 2 min $45 \mathrm{~s}$ : (j) the CG method, (k) the MP method, (l) the wavelet based sparsity method. 


\section{CONCLUSIONS}

Imaging is an important factor in the development of thermal therapies. We showed that transmission tomography is a very promising modality for thermal therapy monitoring. The range of temperatures above zero can be successfully monitored with the methods used in common breast screening. Due to the strong attenuation of the acoustic signal that passes through the frozen tissue, monitoring subzero temperatures is a more difficult problem. The strong attenuation can be efficiently overcome using beamforming techniques. Because the region of interest is insonified from many directions in transmission tomography the shadowing effect is much less noticeable compared to the B-mode scans. Also, the shadowing effect is visible only when the signal passes through two iceballs, that is, along the lines that connect two iceballs. This is because the signal that passes through two iceballs is strongly attenuated. Still, we were able to follow the growth of the 3 iceballs, that was indicated by the sound speed estimation, and confirmed visually. The dramatic change in the sound speed, which is almost a doubling between the unfrozen and frozen tissue enables the imaging of the frozen region with extremely high contrast.

Towards the real time application, we need to reduce the complexity of the reconstruction algorithm. We propose to alternate between the full (usual) and the local reconstruction of the region of interest. In the local reconstruction phase, we monitor only a small region of interest, i.e. the ablation region, and assume that the rest does not change. Still, we periodically perform the full reconstruction in order to correct for possible errors due to the patient movement and global change of temperature.

Inexpensive and easy to use ultrasound tomography has the potential to be the leading modality for thermal temperature monitoring.

\section{REFERENCES}

[1] Onik, G., Cooper, C., Goldenberg, H. I., Moss, A. A., Rubinsky, B., and Christianson, M., "Ultrasonic characteristics of frozen liver," Cryobiology 21, 321-328 (1984).

[2] Rajagopalan, B., Greenleaf, J. F., Thomas, P. J., Johnson, S. A., and Bahn, R. C., "Variation of acoustic speed with temperature in various excised human tissues studied by ultrasound computerized tomography," in [Ultrasonic Tissue Characterization II], Linzer, M., ed., (525), 227-233 (1979).

[3] Johnson, S. A., Christensen, D. A., Johnson, C. C., Greenleaf, J. F., and Rajagopalan, B., "Non-intrusive measurement of microwave and ultrasound-induced hyperthermia by acoustic temperature tomography," in [Ultrasonics Symposium Proceedings, IEEE Cat. T77Ch1264-1SU], (1977).

[4] Laugier, P., Laplace, E., Lefaix, J.-L., and Berger, G., "In vivo results with a new device for ultrasonic monitoring of pig skin cryosurgery: The echographic cryoprobe," Journal of Investigative Dermatology 111, 314-319 (1998).

[5] Gage, A. A. and Baust, J., "Mechanisms of tissue injury in cryosurgery," Cryobiology 37(3), 171-186 (1998).

[6] Littrup, P. J., Freeman-Gibb, L., Andea, A., White, M., Amerikia, K. C., Bouwman, D., Harb, T., and Sakr, W., "Cryotherapy for breast fibroadenomas," Radiology 234, 63-72 (2005).

[7] Duric, N., Littrup, P., Poulo, L., Babkin, A., Holsapple, E., Rama, O., and Glide, C., "Detection of breast cancer with ultrasound tomography: First results with the computed ultrasound risk evaluation (CURE) prototype," Medical Physics 2(34), 773-785 (2007).

[8] Jovanovic, I., Inverse Problems in Acoustic Tomography: Theory and Applications, PhD thesis, EPFL, Lausanne, Switzerland (2008).

[9] Jovanović, I., Hormati, A., Vetterli, M., and Roy, O., "Acoustic tomography apparatus and method." US provisional Patent Application 61/080,358 (July 2008).

[10] Mallat, S. and Zhang., Z., "Matching pursuit with time-frequency dictionaries," IEEE Transactions on Signal Processing 41, 3397-3415 (Dec. 1993).

[11] Lustig, M., Donoho, D., and Pauly, J. M., "Sparse MRI: The application of compressed sensing for rapid MR imaging," Magnetic Resonance in Medicine 58(6), 1182 - 1195 (2007). 